\title{
El Cordobazo antes de El Cordobazo. La vigilancia policial a los estudiantes en mayo de 1969
}

\section{Julieta Sahade}

Programa de gestión y preservación de archivos de la Comisión Provincial por la Memoria, Argentina archivo@comisionporlamemoria.org

Virginia Sampietro

Programa de gestión y preservación de archivos de la Comisión Provincial por la Memoria, Argentina archivo@comisionporlamemoria.org

\section{Ingrid Jaschek}

Programa de gestión y preservación de archivos de la Comisión Provincial por la Memoria, Argentina archivo@comisionporlamemoria.org

\section{Magdalena Lanteri}

Programa de gestión y preservación de archivos de la Comisión Provincial por la Memoria, Argentina archivo@comisionporlamemoria.org

\section{Esteban Soler}

Programa de gestión y preservación de archivos de la Comisión Provincial por la Memoria, Argentina archivo@comisionporlamemoria.org

\section{Soledad Basterra}

Programa de gestión y preservación de archivos de la Comisión Provincial por la Memoria, Argentina archivo@comisionporlamemoria.org

\section{Laura Albañir}

Programa de gestión y preservación de archivos de la Comisión Provincial por la Memoria, Argentina archivo@comisionporlamemoria.org

\section{INTRODUCCIÓN}

Los asesinatos de los estudiantes universitarios Cabral y Bello durante las protestas ocurridas a mediados de mayo de 1969 fueron un punto de inflexión en las crecientes manifestaciones estudiantiles que, unidas a las de otros sectores, desembocarían el 29 de mayo en el Cordobazo. Esto intensificó el funcionamiento del aparato represivo del Estado, respaldado por el andamiaje meticuloso y sincronizado del espionaje de inteligencia en todo el territorio nacional. En la provincia de Buenos Aires, la Dirección de Inteligencia de la Policía (DIPPBA) fue la encargada de controlar e informar todos los movimientos y protestas de los estudiantes, especialmente en las universidades de La Plata, Mar del Plata y Bahía Blanca. El archivo de inteligencia policial -cerrado en 1998 y actualmente gestionado por la Comisión por la Memoria de la provincia de Buenos Aires- muestra a través de miles de fojas un pormenorizado relato de lo que sucedía en el territorio bonaerense, como un preámbulo inevitable de las revueltas estudiantiles y de trabajadores que significaron el comienzo del fin del gobierno de Onganía. 
La información de los acontecimientos que se producirán antes, durante y después del Cordobazo, está reunida en el Legajo 15369 de la Mesa Referencia. ${ }^{1}$ Comienza el día 16 de mayo ${ }^{2}$ y a lo largo de 15 tomos con 2400 fojas se puede advertir fundamentalmente la inteligencia previa al 29 de mayo dentro del territorio provincial así como la relación, mediante requerimientos e informes, establecida con la comunidad informativa a nivel nacional. Miles de fojas incorporadas al legajo minuto a minuto, documentos desprolijos, sin tiempo para editar ni pasar por la Olivetti, dan cuenta del clima caliente, de preocupación y alerta.

Proponemos recorrer esos días previos a partir de la mirada de la DIPPBA; seleccionaremos algunos documentos que permitirán analizar distintos registros de inteligencia y burocracia estatal.

El 15 de mayo de 1969 es asesinado por la policía de Corrientes Juan José Cabral, un estudiante de medicina de 22 años, durante la represión a las protestas estudiantiles por el aumento de los precios en el comedor universitario.

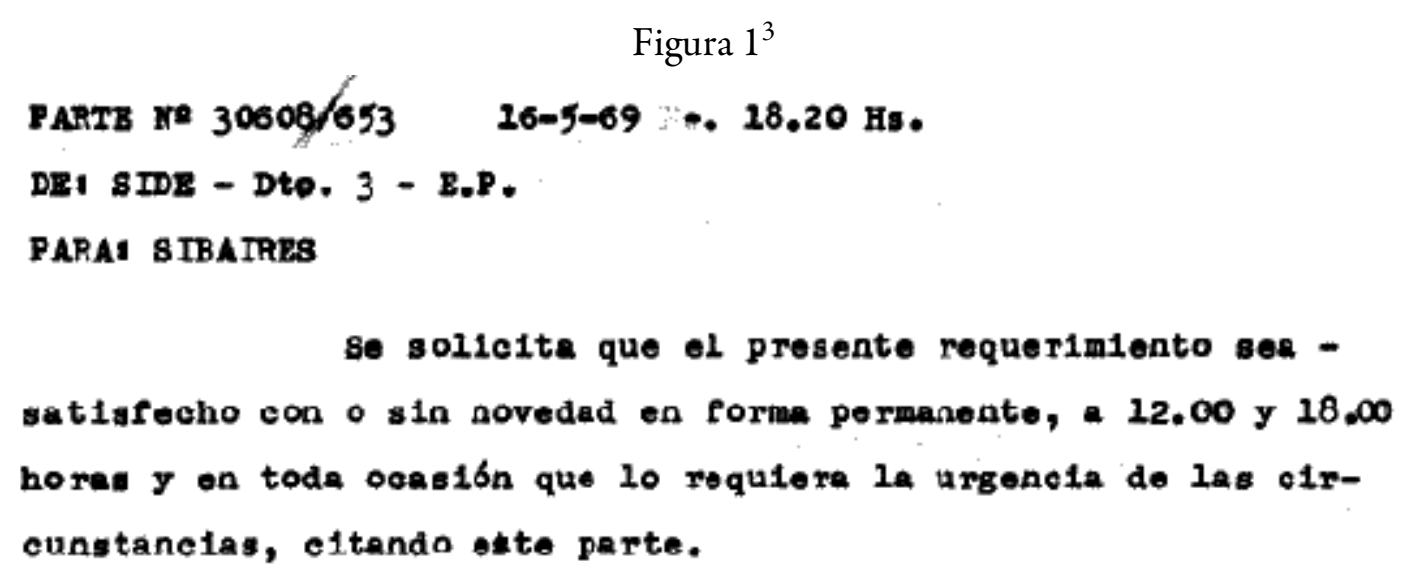

Menos de 24 horas después la Secretaría de Inteligencia del Estado (SIDE) emite el parte número $30608 / 653$ a todos los organismos de inteligencia provinciales para que informen dos veces por día (a las 12 y a las 18 hs, "haya o no novedades al respecto") la situación en el ámbito universitario. A través de este requerimiento la SIDE solicitaba información sobre "actividad de organizaciones marxistas, personas que activan en el ámbito universitario de origen marxista, actividad panfletaria, comunicados de prensa de las organizaciones, rol de las autoridades universitarias; actos realizados en los recintos y fuera de ellos (...) identificación de las personas que toman parte activa en los hechos".

Estos panoramas informativos eran acompañados por panfletos y otros documentos producidos por las distintas agrupaciones estudiantiles.

Figura 2

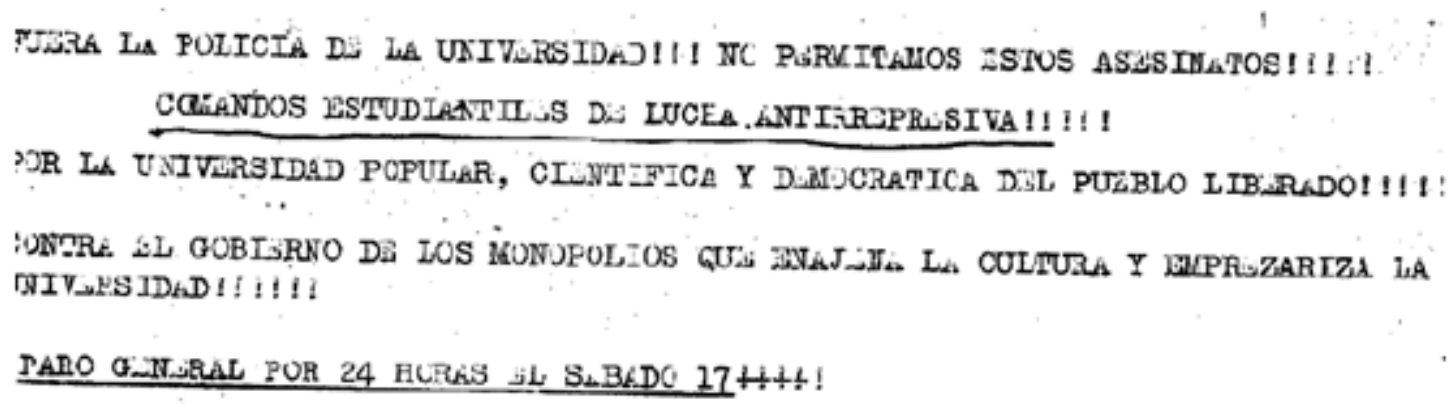

Antes de que se produzca el asesinato del estudiante Adolfo Bello en Rosario, el día 17, la DIPPBA ya había producido varios informes que daban cuenta de las acciones de los estudiantes en las universidades de La Plata, Bahía Blanca y Mar del Plata. Después de la muerte de Bello, los partes informando la situación serán más frecuentes. 
Figura 3

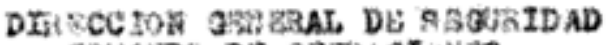

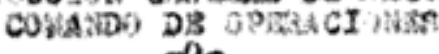

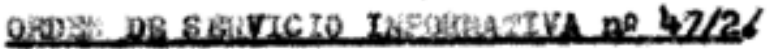

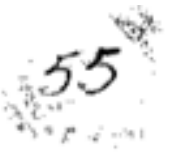

\author{
seior Jefe de policfes
}

Con notaro de in eridente dis pos ición de grupos estudiant1les para realizar acciones diversas durante la sozians entranto $y$ haborse agravado al hocho prinero al groducis. se el deceso del estadiante CobOlFO BELLO, on la focha so amplia

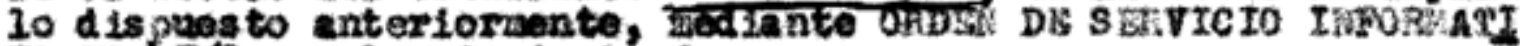
7/A $1047 / 2$, on 12 siguiente format

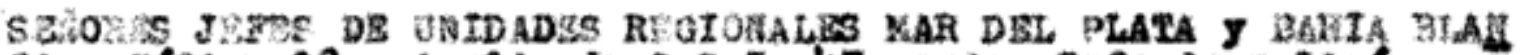
CA. - CSA1 180 - Ampliando 0.S.I. 47, orden Jefe de olicla, ma tivo repeticion necho CIUDAD $\mathrm{KOSARIO}$, previntendo alteraciones

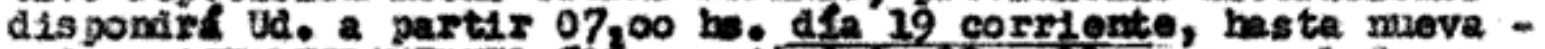

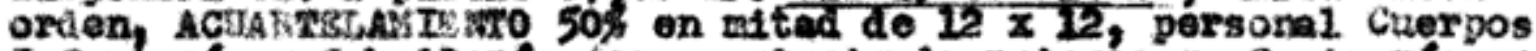
Infanterfa y Cabalierfo baa, minteniendo rotenos on Condsarfas $\theta$ sa cludad. Intergedio Dele gación S. T.?.B.A y personal Irvastige clones, asegurar informelones finos preventr. Personal so bantondre aprestedo prefezentemente egrupados cuerteice, salvo oraIuación Inil que necesidad tenorios lugnres estratéricos, dobión-

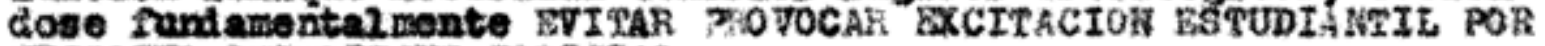
IXCSTTV DES LIBOUB POLICIAL.

El Legajo 15369 reúne partes de inteligencia de distintos organismos, requerimientos, memorándums, telepartes, volantes, documentos políticos, panfletos y recortes periodísticos que relevan información de gran parte del territorio nacional. Uno de los aspectos tal vez más llamativos del legajo, además de la premura con la que se advierte fue constituyéndose, es la existencia de directivas referidas a medidas represivas, aspecto que no suele hacerse presente en legajos de inteligencia.

La posibilidad de que la agitación social se exceda de los marcos de la vida universitaria inquieta a los servicios de inteligencia. El día 19 de mayo, la SIDE comparte su apreciación del panorama universitario:

Figura 4

"in afntenins on Ia counma del 18 al 25 de mago

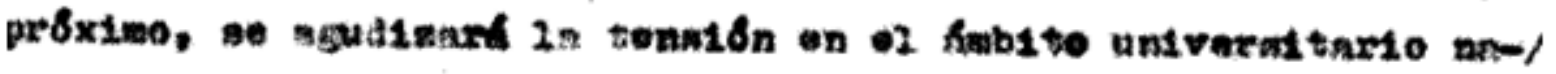

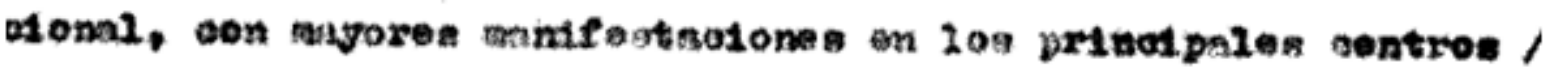

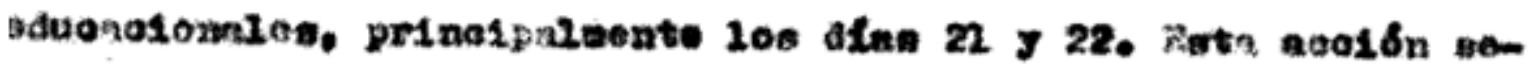

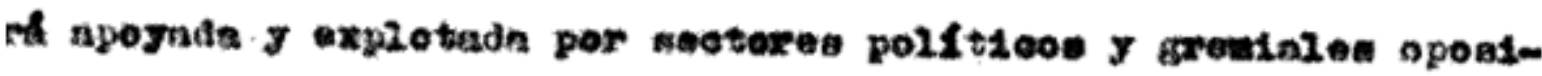

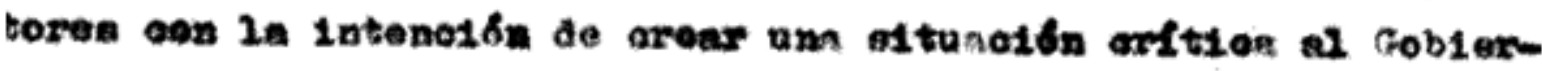

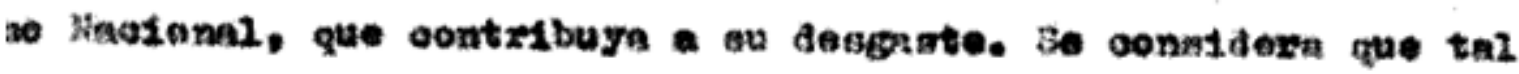
ujiraction no no verk oonoretada, por no atar dadav las oona1-/ tones para ax.20". 
A partir del lunes 19 de mayo se suceden telepartes, informes de inteligencia locales (Mar del Plata, Bahía Blanca y La Plata) volantes y documentos de las agrupaciones convocando a marchas, manifestaciones y a la huelga. Los primeros intentos de sumar a otros sectores no tienen aún mayor eco en la ciudad de La Plata. Luego de informar que un grupo de estudiantes de la UNLP se acercó a la sede de la CGT de los Argentinos a pedir la solidaridad y participación en la marcha del silencio sin mayor éxito, resaltan la respuesta de la Unión Ferroviaria:

Figura 5

\section{Ia única excepoión a esta actitud,fué puesta de manifiea- to por la UNION FERROVIARIA(Seccional Tolosa), la que se expreab́ en el sentido de solidarizarse con el estudiantado universitario del paí, y asegurar eu partioipación aotiva en el acto referido}

El martes 20 de mayo se llevaron adelante actos y manifestaciones en las distintas universidades y se anticipa la huelga estudiantil nacional del miércoles 21. Comienzan a intervenir nuevos actores en el escenario: las autoridades de la UNLP, UNS y UNMdP dan asueto y estudiantes secundarios de varias localidades -entre ellas Bahía Blanca, San Nicolás y Pergamino- adhieren a la medida de fuerza.

Figura 6

TE SENTIRAS AJ

HAS PBNSAD OUB TU OOLABORACI^N ES INDIBPBNBABLB?

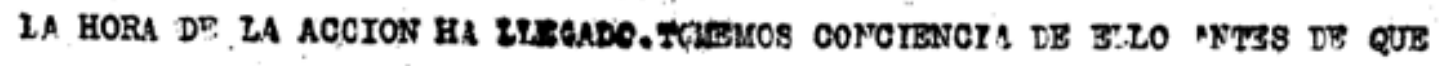
JEA JELASIND TREDE.

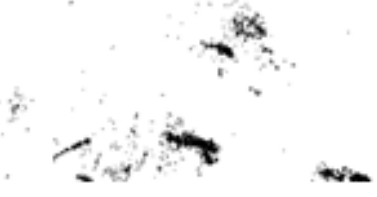

ALTMRNOB DEL COLBGTO NACTONAL Y
ANBXO COIGECTO - TURNO NOCHE -

La prensa comienza a dar cuenta del clima de agitación obrero estudiantil: manifestaciones callejeras, toma de facultades, asambleas, reuniones entre agrupaciones estudiantiles, obreras y políticas; pronunciamientos públicos y convocatoria a la huelga. La frecuencia de las comunicaciones entre los diferentes organismos de inteligencia y las consignas esgrimidas por los estudiantes en volantes, proclamas, pintadas en la vía pública y cánticos callejeros son reveladores de una aceleración de la dinámica política de los actores.

Los servicios de inteligencia y de seguridad redoblan el alerta y la Dirección general de Seguridad -comando de operaciones- emite la Orden de servicio informativa número 48 donde se imparten directivas de cómo actuar frente a la creciente agitación estudiantil que empieza a regir en todo el territorio provincial y nacional. 
Figura 7

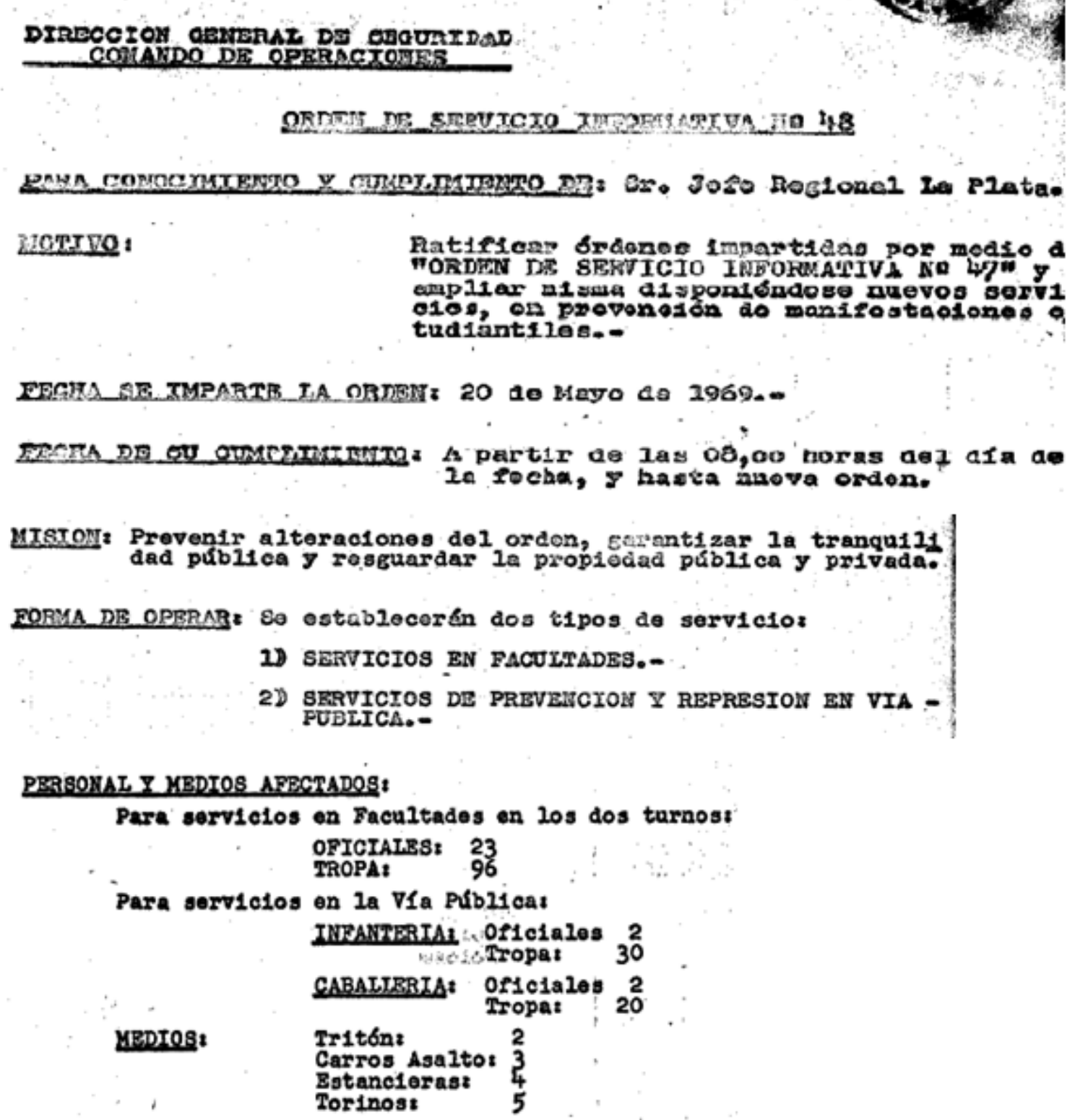

En la jornada del 21 se multiplican las acciones de protesta en las ciudades universitarias. La aplicación del "plan de prevención de manifestaciones estudiantiles" deja como saldo numerosas detenciones. 
Figura 8

ASUNTO: InP. G/ PANUZALA UNIVETIIÁRIO - Ampliación -

24,00 Siendo lu sora indicada al targen, 108 estudinntes universitarios, continúan en la zona centrica compren. dida entre las calles 7 a 8 y de 46 a 51 , arrojando en forma constarte gran cantidad de petardos de regular poder. Los misioos pasn practicamente desapercibidos, en razon de la presencia de simpatizantes del. Club lstudiantes, que se encuentran festejando la con quista de la nueva Copa de Anérica.Se adjuntan panfletos gue fueran arrojados.DMPARANADO BUSQUEDA, Wayo 21 de 1969.-

Figura 9

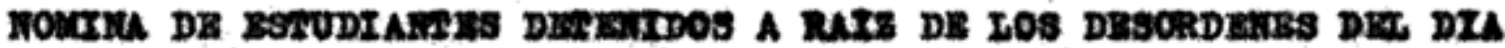

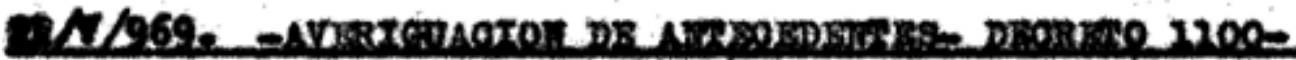

El día 22 de mayo la Dirección General de Seguridad declara a Rosario "Zona de emergencia”. Ese mismo día, inspectores de la Brigada de Investigaciones de San Nicolás van a Rosario y se entrevistan con funcionarios de la policía de Santa Fe y de la Policía Federal para interiorizarse de la situación del "conflicto estudiantil" y su impacto en otros actores sociales.

Figura 10

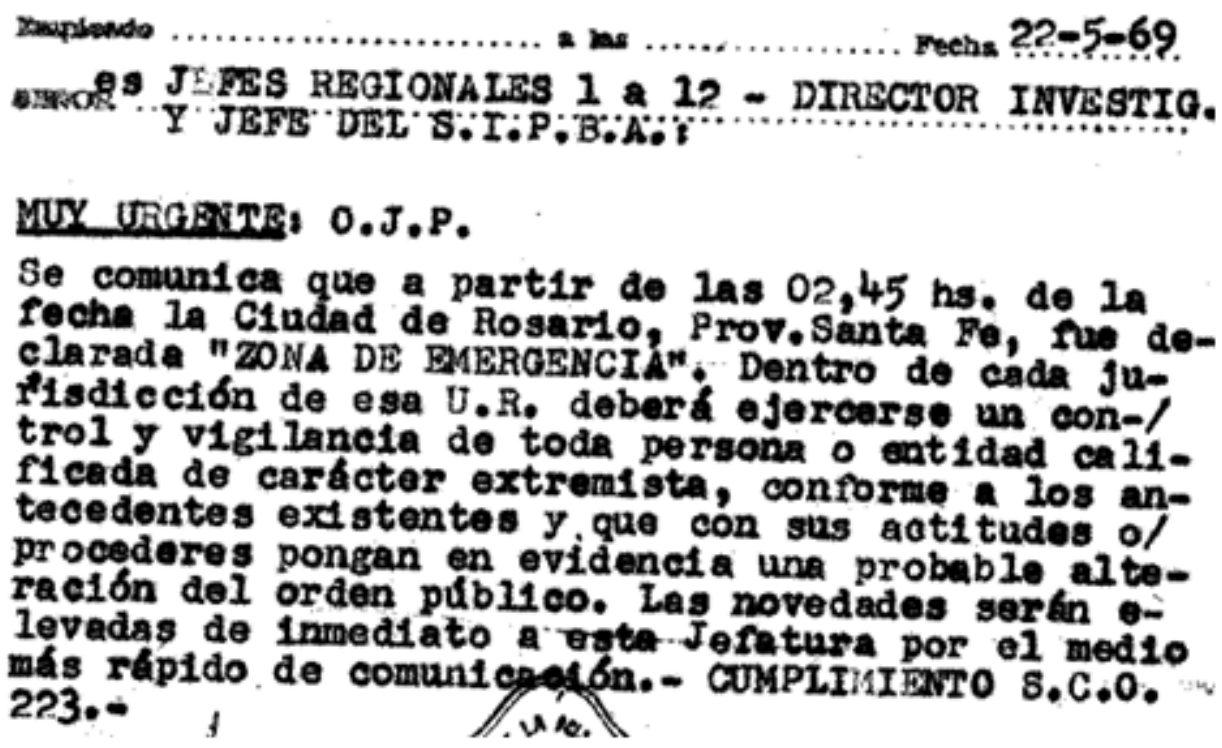


El viernes 23 se desarrollan numerosas reuniones, actos y manifestaciones en Bahía Blanca y La Plata. Numerosos panfletos son recogidos por los agentes de la DIPPBA.

Figura 11

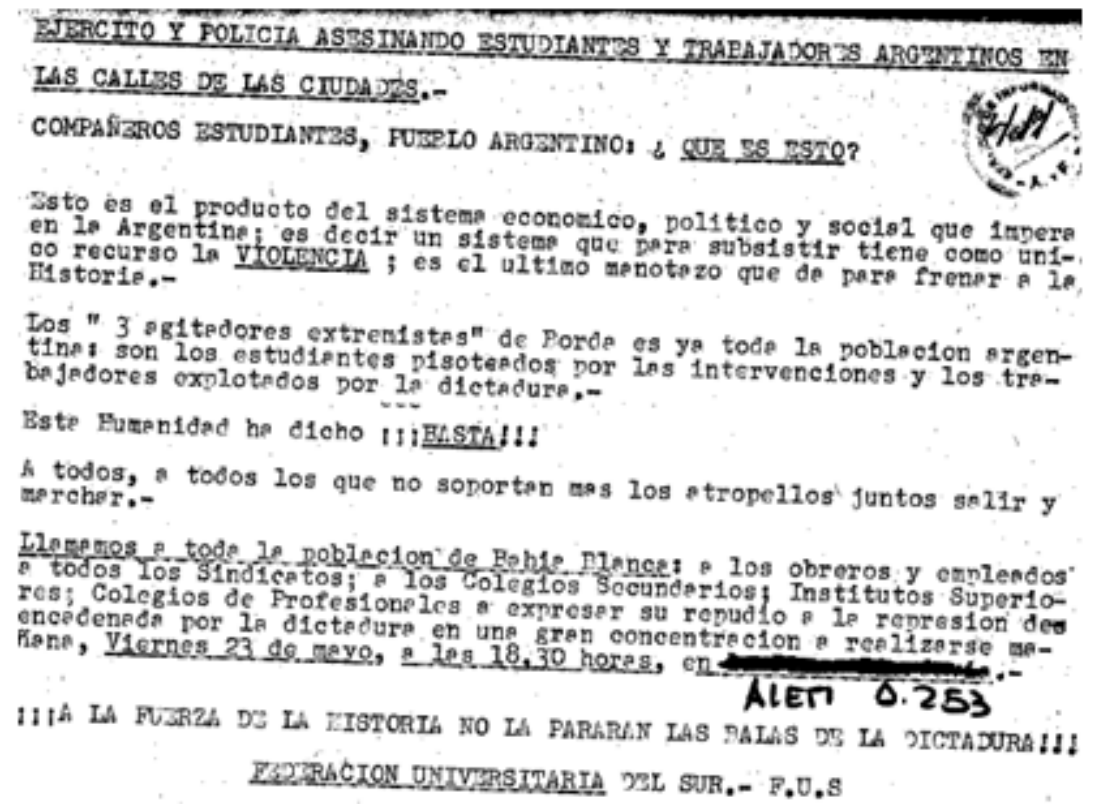

Después del fin de semana en el que se destacan las manifestaciones estudiantiles en Mar de Plata durante el desfile militar por el 25 de mayo, el inicio de la semana marca la profundización de las consignas y las acciones. Podemos identificar desde los reclamos por las detenciones perpetradas la semana anterior hasta las expresiones contra la dictadura. Las convocatorias invitan a los distintos sectores de la sociedad a confluir en una jornada de lucha obrero estudiantil. En La Plata, la FULP llama al paro obrero estudiantily popular el día 29 de mayo, bajo la consigna "La lucha de los estudiantes es la lucha de todo el pueblo"

Figura 12

\begin{abstract}
Bi jueves ha de ser jornada oombativa donde golpeemos juntos a la diotadura y profundioemos atn más este prinoipio de su orisis general.

CONRA IA DICTADURA AMI POPULAR, BNTRBOUISTA Y EXPLOTADORA, FIEL REPRBSEMTAMTE DE LAA DEPENDENCIA BCONOMICA Y LOS INIMRRBSES DBL IMPFRIALISNO. POR UNA.SALIDA QUE BXPULSE AL IMPERIALIBANO Y LIQUIDE AL 'LATIFURDIO ( $4 \%$ de los propietario posee el $54 \%$ de la tierra ovltivable).

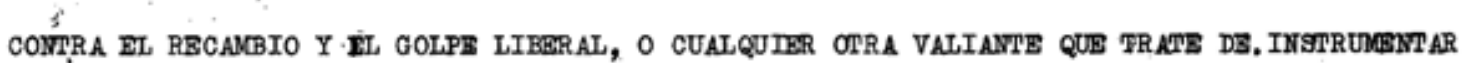
NUESTRAS LUCHAS PARA DAR UNA SALIDA QUS, CACBFANDO BL GOBIBRNO, DBJS IMTAGTO BL SISTEMA ECONOMGCO-SOCIAL DEPENIEMTE DE LOS YONOPOLIOS EXTRANJBROS.

?

CQYYRA LA RBPRESION, COMRA LA INVASION MIHITAR Y LA LEY MARCIAL BNT ROSARIO. POR EA ORGANIZACION DE LA RESISTEMCIA Y BL BAEREAT AUCBMTO POPULAR.
\end{abstract}

CONTRA BL LIMITACIONISUO, BL AUTORITARISMO Y LA BXSENIANZA DESTINADA A FABRICAR BJECUMIVOS, TECNICOS $\mathbf{3}$ IDEOLOOS QUE SOPORTEN Y JUST IFIQUEN EL SISTEMA ACTUA. POR UNA UNIVERSIDAD DEMO-. CRATICA AL SEVICTO DE LA CLASE OBRERA Y DEL PUDBLO.

El 27 de mayo, la SIDE informa el panorama en Tucumán. 
Figura 13

D :SID (C NT.ICIA)

PARA :SI - SIA-S̈IN-SIGN -PR F GTURA - -COUROIMUCIUN F D.SIPBA.-

SE HA AGUUIZADO LA SITUACION ESTUDIANTIL EN LA CIUDAO DE TUCUMAN DONOE LOS STUDIANTES MAN PRODUCIDO DESMANLS (ROTURA DE PULRTAS $Y$ VENTAMAS DE CASAS E INCENDIANDO aUtOMOVILES). La POLICIA Ha TRATAdo De RÉPRIMIR en GRICMMENTEA LOS REVOLTOSOS Pë̈ro SU INTERVENCION // PUEDE SER desBordada POR ESTOS. ANTE TAL CIRCUNSTANCIA El gOBERM DOR DE LA PROVINCIA SE HA PUEST̈O EN CONTACTO CON QL MINISTRO DE INTERIOR Y P DIRLA+++PEDIRIA LA INTERVENCION DEL EJERCITO.

EL COMANDO DE LA STA.BRIGADA ORDENO LL AUARTELAMK NTO"D LAS TROPAS. -

El 29 de mayo, la DIPPBA se encuentra abocada a la producción y reunión de información sobre el paro dispuesto para esa jornada. A partir de media mañana empieza a recibir telepartes reservados de la SIDE, con carácter de "Urgente". Llegan noticias desde Córdoba:

Figura 14

CORDOBA:

- SIENDO UAS 1015 HORAS LA CIUDAD PRESENTA EL SIGUIE NTE CUAOROGC - IMCTIVIDAD TOTAL EN LAS DOS UNIVERSIDADES ( OFICIAL Y CATOLICA)

IMASA ESTUDIANTILES MANTNIA TRANQUILA.

$1=0 x$ :

- piquetes de la hullga de alumnos secunoarios recorren la ciudad aRROSANDO PANFLETOS.

B) AMBITO GREMIAL. .

- obretros dé iKa AB̈andonaron pacificamente ule su trabajo.

- Pa o++ PERSONal de energia y combustible abandonan su traB̈ajo.

UN GRUPO DE 200 PERSOMUS AL PARECER DE LUZ Y FUERZA Y CGT

REALIZAN MANIFESTACIONGS ARROJANDO BOMBAS DEESTRUENDU EN EL LENTRU dE LA CIUDAD. La POLICIA DISUELVE CUN gaSES.

LOO HURAS : UN GRUPÖ DE MAWTESTANTË̈ COMEWTEN DESMANES EN EL CENTRO INCENDIANDO DOS O TRES VGHICULOS. MIENTRE QUE E. OTROS PROCEDIBRON A LA COA CLAUSURA DELOS PUENTES

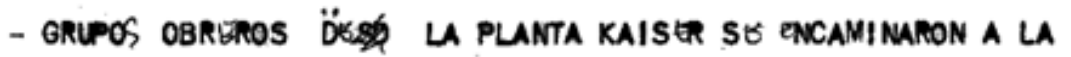
CIIUDAD SIONDO INTERCEPTADOS POR PERSONAL DE LA GUARDIA DE INFANTARIA DE LA POLICIA FGDERAL, FRENTE A LA PLAZA LA PAZ.

A LOS MISMOS SE LE UNÍRON ESOUDIANTIS BE +5 QUE AGURDABAN̈ EN LAS IMMEDIACIONES, APEDRETMDO LOS CONERCIOS DELA ZONA CONTRICA Y DESTRUYG UMUS 200 VIDRIERAS. S EARROJARON BOMBAS MOLOTOV LAS QUE PROVOCARON UN PRINCIPIO ÖE INC WOIO, DE DOS COMERCIOS. 
La contundencia de los hechos ocurridos en Córdoba el 29 de mayo de 1969 ha desdibujado de la memoria colectiva el clima y los sucesos previos ocurridos a nivel nacional. El legajo de Referencia 15369 amplia el foco temporal y geográfico, permitiendo mirar en perspectiva y analizar la participación en otros territorios, en el proceso de creciente movilización y represión que desemboca en el Cordobazo.

\section{Notas}

1 El fondo de la DIPPBA tiene una estructura que clasifica y agrupa la documentación en "mesas", y hacia el interior de las mesas, "factores", "carpetas" y "legajos". En cierta medida la estructura del archivo es reveladora de los sujetos sociales, organizaciones y actividades sobre los que la DIPPBA detenía su mirada. La documentación relacionada con las universidades se encuentra mayoritariamente en la mesa A, factor estudiantil. Sin embargo, la información sobre el Cordobazo está en la Mesa Referencia, que tiene la particularidad de agrupar legajos para todo aquello que no podía ser clasificado en alguna de las otras mesas, o bien para aquello que les interesaba a todas. Podría decirse que es un punto de cruce con el resto de las mesas. La mayoría de los legajos son temáticos y hay algunos personales.

2 El legajo finaliza en agosto de 1984, con las repercusiones de los actos conmemorativos del Cordobazo. Los tomos 2 a 7 reúnen recortes periodísticos de los acontecimientos que llevarían al Cordobazo en diferentes provincias.

3 Todos los fragmentos de documentos seleccionados para este trabajo están incluidos en el legajo: CPM, Fondo DIPPBA, Div: Cen. A y F, Mesa Referencia, Legajo 15369, Tomo I Rubro I y Tomo I Rubro II 